\section{HOW LONG IS A PIECE OF STRING?}

Or there again, how far should an umbilical catheter be inserted to land on the sweet point in the vein or artery respectively? The system based on linear measurements which remains in common use was published by Peter Dunn 50 years ago; it was derived from post-mortem studies so it had high face validity, but was never to my knowledge subjected to a rigorous comparison with any other method. Filling this gap in knowledge, Kieran et al have done a randomised comparison of the linear measurement method against a formula based on birth weight: they found that using weight did not improve the accuracy of placing umbilical venous catheters, compared to linear measurement, but was significantly better with respect to arterial catheters. This is a really useful, practical result which could be incorporated in guidance in every neonatal service and would be a neat piece of quality improvement. See page F10

\section{BURSTING THE BUBBLE}

Over thirty years ago, my first introduction to continuous positive airway pressure support was 'bubble CPAP': a crude but effective and very cheap means of delivering CPAP, in which the pressure applied to the baby's airway is variable by means of the depth of a water seal in the expiratory arm of the gas delivery system. Nowadays we use flow drivers with fluidic valves to ensure maintenance of airway pressure by enhancing flow during inspiration, and there has long been good evidence that this modality is in general better than plain CPAP. However these studies were all done in modern intensive care settings in developed countries. Mazmanyan et al report an equivalence trial of bubble versus flow driver CPAP in Armenia, in a setting without either blood gas analysis or surfactant. After stratification for sex and antenatal steroids, there were 59 and 66 babies randomised to the flow driver and bubble arms respectively, and on the primary outcome, days of treatment, bubble was equivalent to flow driver. But astute readers will notice that on aggregate there were over twice as many adverse secondary outcomes in the bubble arm, so you will have to decide for yourselves whether you agree that the two treatments are really 'equivalent'. See page F16

\section{Q METHODOLOGY AND ATTITUDES TO CARE}

Admittedly, 'Q methodology' sounds either like fake science or the title of a pacy thriller, but in the context of the paper by Gallagher et al it means something completely different: a way of quantifying attitudes, then applying this method to consultants and trainees in neonatal medicine in relation to considering treatment options for extremely preterm births. Attitudes to, and perceptions of the risk of death or disability are likely to impact both on the content of discussions with parents and the enthusiasm with which resuscitation and subsequent care might be conducted. The results cannot be summarised in a sentence so I urge you to read this important paper carefully. It goes without saying that many physician attitudes are culturally determined: the adjacent paper by Chan et al provides a complementary perspective from Hong Kong, so the two papers really need to be read together. The next step, long overdue in neonatal care (though used for the last 40 years in general practice research), will be to do content analysis of recorded parental discussions. Then at last we will truly understand first, what we are really saying to parents; second, how we are saying it; and finally, and even more important - do we ever listen to parents rather than just talk at them? See pages F31 and F37

\section{GRAM NEGATIVE INFECTIONS}

The new neonatal unit in Glasgow was the recipient of unwelcome publicity in November 2015 when several babies became colonised with Serratia marcescens, and one reportedly became septicaemic and died. So the review by Stapleton et al is timely: outbreaks of infection with resistant gram negative gut organisms like Serratia can happen in any unit at any time, and the underlying cause is often not discernible. Several other major points emerged from the review. First, these outbreaks can be very disruptive, with a median duration of over 6 months. Second, the mortality among infected babies was high at 31\%. Finally, understaffing and overcrowding were identified as common contributory factors. As the evidence continues to build that adequate numbers of appropriately skilled nurses are central to both safety and quality in neonatal care, this paper adds another important strand to the argument. See page F72

\section{MATERNAL DIET AND FETAL OUTCOME}

The cliché 'you are what you eat' has always been important but never more so in an age when obesity is epidemic. In the November edition of Fetal \& Neonatal we carried a paper linking maternal smoking and poor diet to the risk of moderately preterm birth. In this edition, Botto et al report an association between improved diet quality and a reduced risk of certain congenital heart defects in a case control design, with just under 10000 subjects in each group, from the National Birth Defects Prevention Study in the USA. A 'better' diet meant a composite of more 'Mediterranean' foodstuffs, together with more nutrients known to be important in pregnancy, such as folate. This method yielded a ten point scale of diet quality against which could be ranked the relative risk of cardiac anomalies. The association between better diet and reduced rates of heart defects was striking for 'all conotruncal defects', tetralogy of Fallot, and secundum type atrial septal defects; but there was no association for ventricular septal defects. See page F43 